\title{
Statistical properties of strongly nonlinear waves within a resonator
}

\author{
V. B. Efimov, ${ }^{1,2, *}$ A. Ganshin, ${ }^{1}$ and P. V. E. McClintock ${ }^{1}$ \\ ${ }^{1}$ Department of Physics, Lancaster University, Lancaster LA1 4YB, United Kingdom \\ ${ }^{2}$ Institute of Solid State Physics RAS, Chernogolovka, Russia \\ (Received 1 September 2008; published 31 December 2008)
}

\begin{abstract}
An experimental investigation of nonlinear waves is reported for a system of one-dimensional second sound waves in superfluid helium within a cylindrical resonator of high $Q$ quality factor. The strong nonlinear dependence of the wave velocity on amplitude distorts the wave shape and leads to the formation of multiple harmonics. The restricted geometry of the resonator results in a discrete energy spectrum, where the energy is transmitted from the driving frequency to the high-frequency edge of the spectrum, where dissipation occurs-a Kolmogorov-like energy distribution. It is found that the main resonance occurs at the driving frequency, and that the next few harmonics are approximately sinusoidal, coherent with the driving force, but that higher harmonics appear to be chaotic and are no longer phase coherent with the drive. For developed turbulence, the probability density function of the high-frequency harmonics is well approximated by a Gaussian distribution. Thus, the nonlinear acoustic waves exhibit the statistical properties distinctive of weak turbulence, confirming that they can properly be treated in terms of a statistical description.
\end{abstract}

DOI: 10.1103/PhysRevE.78.066611 PACS number(s): 43.25.+y, 67.25.dk, 47.27.-i, 47.35.Rs

\section{INTRODUCTION}

Turbulence is a far-from-equilibrium state of a nonlinear physical system whose energy distribution extends over many degrees of freedom. The most important physical process in turbulence is that of energy transformation to heat, via dissipation, starting from the form of energy that is being pumped into the system from an external source. The turbulence behavior may be defined by the physical nature of the processes providing the energy pumping and energy transformation. In this sense we may distinguish between kinetic, convectional, and acoustic turbulence. These are described by similar nonlinear equations, but their driving and inertial forces are different in nature, and there are different reasons for the instabilities in the motion of the media, and different processes of energy transformation. In each case, however, the dissipative process is the viscosity of the medium. Kinetic turbulence in a moving fluid appears when the inertial effects of motion are greatly dominant over viscous forcesthe situation of high Reynolds numbers. Convective turbulence appears for steady heating of a liquid in a gravitational field, when the ratio between the Archimedean and viscous forces becomes large (high Rayleigh numbers). A similar situation arises in a medium for which with the dependence of wave velocity on amplitude becomes nonlinear for waves of large amplitude. Nonlinear effects then dominate over dissipative processes (high acoustic Reynolds numbers). This is the case of acoustic turbulence. It is characterized by steepening of the wave at either front or back, leading to the formation of wave breakdown and shock waves and to the creation of high-frequency harmonics of an initially harmonic driving force.

For turbulence in an incompressible liquid, for example, kinetic energy is pumped in through external mechanical forcing and is finally dissipated by viscosity. As suggested by

\footnotetext{
*victor_efimov@yahoo.co.uk
}

Richardson [1], the kinetic energy at first flows in a dissipation-free manner towards successively smaller length scales. This is called the inertial interval. The cascadelike energy transfer process is eventually terminated by viscous damping, once the Reynolds number approaches unity. On each length scale it is possible to attribute a frequency to the corresponding excitation mode.

The process of energy transformation through the inertial interval is dependent on interactions between the different modes. These are described as weak, giving rise to weak turbulence, in cases when the energy exchange $\Delta E$ in the interaction process is much less than the energy $E$ of each mode. Strong turbulence is the opposite case, where $\Delta E$ $\sim E$.

From a theoretical point of view, the simplest case is the turbulence of weakly interacting waves, when there is strong dispersion of waves in the medium but the mutual interactions of the wave packets are weak, and the phases of the different waves are consequently random $[2,3]$. This approximation allows us to develop a statistical description for a very wide class of pumping forces. The cascade idea explains the basic macroscopic manifestation of turbulence: The mean rate of viscous energy dissipation does not depend on viscosity at large Reynolds numbers.

The statistics of the velocity fluctuation distribution in turbulent flows was quantified by Kolmogorov [4,5], who derived the " $-5 / 3$ law" for the energy spectrum at intermediate scales $E_{k} \sim k^{-5 / 3}$, within the inertial interval of high Reynolds number flows. In the ideal case, the energy density is homogeneous (statistically invariant under translation) and isotropic (statistically invariant under rotation and reflection) in the three Cartesian velocity dimensions. One-dimensional weak turbulence with a constant energy flux $P$ is described by the relationship [6]

$$
E_{k} \sim P^{1 / 3} k^{-5 / 2}
$$

in the case of $\omega_{k}=|k|^{a}$ for $a>1$, where processes of the type $\omega_{k} \leftrightarrow \omega_{k_{1}}+\omega_{k_{2}}+\omega_{k_{3}}$ are significant, amounting to say $>15 \%$ 
of all interactions. They result in a stationary energy flux to the high-frequency edge of the spectrum where dissipation occurs. The wave amplitude of weak turbulence in the inertial interval is expected to exhibit statistics close to Gaussian.

One example of weak turbulence is the interaction of waves on the surface of a fluid $[7,8]$. The turbulent nature of the surface waves is very close to the case of acoustic turbulence-nonlinear dispersive waves propagate along the fluid surface and form a weak, interacting wave packet subject to negligible dissipation. The statistical behavior of a surface wave can be described within the framework of the random wave theory [9].

Strong turbulence corresponds to the case where the assumption of weak interaction between harmonic waves does not apply. The time of interaction between different wave modes is then comparable to the time of wave propagation and the waves are cophasal. Intense acoustic waves in media with high nonlinearity, weak dissipation, and weak (or absent) dispersion provide typical examples of strong turbulence $[10,11]$. The propagation of dispersionless nonlinear waves is described by Burgers' equation [12] and in a onedimensional geometry can be written as

$$
\frac{d u}{d t}+u \frac{d u}{d x}=\nu \frac{d^{2} u}{d x^{2}},
$$

where $\nu$ is the coefficient of dissipation. Strong interactions between the harmonics leads to wave disturbance and the formation of shock waves [13]. The origin of the traveling waves is the same-some external force, which in the case of second sound is a periodic heating by selected shape of signal from a generator. Theoretical investigation of the randomly forced Burgers' equation has shown [14] that the velocity gradient should have a probability density function (PDF) with a power-law dependence on the velocity gradient. If the system is excited by noise, a statistically average random signal is to be expected, which subsequently transforms due to the nonlinearity in the wave velocity. Higher pulses merge with smaller ones during the process of wave propagation [15]. This reformation drastically changes the statistically average random distribution to a nonequilibrium one.

Any harmonic wave launched into a medium with a nonlinear dependence of wave velocity on amplitude must eventually transform towards sawtooth waves. Its main dissipative processes arise though the formation of shocks [16], with dissipation acting to smooth the breakdown of the wave. The extreme case in the transformation of a harmonic nonlinear wave is a sawtooth shaped wave

$$
\left.u(t)\right|_{0} ^{2 \pi}=u_{0}+\sum_{n=1}^{\infty} \frac{1}{n \pi} \sin (n \omega t) .
$$

The energy spectrum of such a wave is similar to the Kolmogorov spectrum $E_{\omega} \sim \omega^{-2}$, though the component harmonics of this wave are cophasal and the PDF is a flat uniform distribution. This distribution differs from the case of weakly interacting wave modes, and the random phases of the sinusoidal modes justifies a statistical approach to the wave interactions.
In this paper we report the results of an experimental study of the statistical behavior of nonlinear second sound waves in superfluid ${ }^{4} \mathrm{He}$ in a quasi-one-dimensional resonator pumped by a harmonic signal. The resonant conditions allow us to raise the amplitude of the standing wave sufficiently to facilitate investigation of the influence of nonlinearity, but without significant disturbance of the medium by the net heat flux from the emitter. The standing wave in the resonator can be described by the sum of two counterpropagating waves periodically pumped by the emitter. Given the strong nonlinearity of the medium, such waves would adopt a sawtooth shape as the limiting case of wave transformation. The PDF of their sum is a nonequilibrium one. In what follows, we study the PDF of such standing waves in a high- $Q$ (quality factor) resonator and try to understand how strongly the different multiple modes in the resonator are mutually correlated.

\section{EXPERIMENTS}

The velocity $c_{2}$ of a second sound wave in He II depends strongly on its amplitude $\delta T$ and can be approximated as

$$
c_{2}=c_{20}(1+\alpha \delta T),
$$

where $c_{20}$ is the wave velocity at negligibly small amplitude, $\delta T$ is the wave amplitude, and $\alpha$ is the nonlinearity coefficient of the second sound velocity.

There are huge advantages in the use of roton second sound waves in He II for model studies of acoustic turbulence. Within the experimentally convenient temperature range $2.17-1.5 \mathrm{~K}$ the nonlinearity coefficient $\alpha$ can be tuned, just by changing the bath temperature: $\alpha \rightarrow-\infty$ near the normal-to-superfluid transition $T_{\lambda}=2.17 \mathrm{~K} ; \alpha$ passes through zero at $T_{\alpha}=1.88 \mathrm{~K}$; and $\alpha \sim+2 \mathrm{~K}^{-1}$ at $T \sim 1.5 \mathrm{~K}$ [17]. Thus one can study the dynamics of both nearly linear and strongly nonlinear waves with positive (such as conventional sound) or negative nonlinearity while using exactly the same experimental techniques. The experiments at $T=T_{\alpha}$ allow us to study the propagation of waves of high intensity for the case of a cubic nonlinearity. Such possibilities are unavailable in conventional experiments. Second sound waves have only a tiny dispersion in the lower frequency range below $1 \mathrm{MHz}$. The fact that the velocity of second sound $u_{20} \leqslant 20 \mathrm{~m} / \mathrm{s}$ is more than an order of magnitude less than the velocity of conventional sound in gases and in condensed media allows us to increase the time resolution of the measurements.

Details of the experimental design have been given elsewhere $[18,19]$ but, briefly, the resonator was formed from a quartz tube of diameter $\sim 15 \mathrm{~mm}$ and length $L=70 \mathrm{~mm}$. Its two ends were capped by a thin film heater and superconducting $\mathrm{Cu}+\mathrm{Sn}$ bolometer [20], respectively. A meander strip heater provided a spatially uniform heat flux. The bolometer had a sensitivity of up to $\sim 10 \mathrm{~V} / \mathrm{K}$. An external magnetic field was used to adjust the temperature of its maximum sensitivity. The heater was driven by an external sinusoidal voltage generator within the frequency range $0.1-100 \mathrm{kHz}$. The frequency of the second sound emitted from the heater (2 times the frequency of the voltage generator) was set 


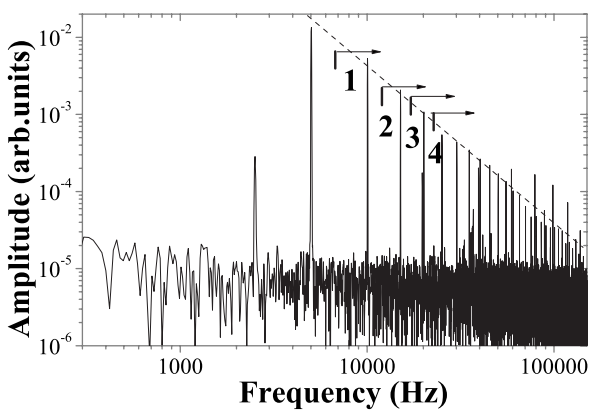

FIG. 1. Power spectrum of a second sound standing wave showing the amplitudes of the multiple harmonics in developed acoustic turbulence. The resonator was pumped at frequency $f_{d}=5030 \mathrm{~Hz}$ corresponding to the 51st resonance; the driving amplitude from the generator was $U_{G}=5 \mathrm{~V}$ at $f_{d} / 2$ (heat flux $W=25 \mathrm{~mW} / \mathrm{cm}^{2}$ ). The arrows indicate the frequencies below which the initial signal was cut by digital filtration. The peak at $f \sim 2.5 \mathrm{kHz}$ is an artifact corresponding to direct electrical pick-up of the driving signal.

equal to one of the longitudinal resonant frequencies of the cavity. It should be borne in mind in what follows that some radial modes may also have been excited due to small nonidealities of the cell construction. The resonance corresponding to the eleventh longitudinal mode was of exceptionally high quality: We note that it coincided closely with the first Bessel solution, and we suspect that this is no coincidence. Thus we do not know the precise nature of the mode excited at the frequency we identify as the eleventh longitudinal resonance.

The $Q$ factor of the resonator, determined from the widths of resonances at small heat flux densities, was $Q \leqslant 1000$ for resonant numbers $p<10$, and $Q \simeq 3000$ for $30<p<100$. The resonance conditions allow the formation of standing waves of high intensity ( $\delta T$ up to a few $\mathrm{mK}$ ) with excitation levels $W \leqslant 50 \mathrm{~mW} / \mathrm{cm}^{2}$. The Mach number can reach $M$ $=\alpha \delta T \sim 10^{-4}-10^{-2}$, and the acoustic Reynolds number, defined [21] as $\operatorname{Re}_{\mathrm{ac}}=\alpha c_{20}(\partial \delta T / \partial x) / \nu \sim \alpha \delta T$, can reach a value up to 100 .

\section{RESULTS AND DISCUSSION}

Given the high $Q$ of the resonator, a sufficiently high sinusoidal drive amplitude leads to the formation of multiple discrete harmonics. Energy is thus transformed, moving from the driving frequency towards the high-frequency edge of the spectrum, where it then dissipates due to viscosity and imperfections of the resonator, i.e., a Kolmogorov-like cascade as shown in Fig. 1. Such behavior is a distinctive feature of turbulent processes.

We determined the statistics of standing waves in the resonator experimentally at $T=2.08 \mathrm{~K}$ by analysis of a developed acoustic cascade with multiple harmonics [22,23]. The main harmonic comes of course from the excitation by the driving signal and is cophasal with the pumping excitation. The driving signal may affect the first few higher harmonics until the mutual interactions of the higher modes deemphasize the influence of the phase of the pumping signal. We therefore used a digital filter to eliminate the lower

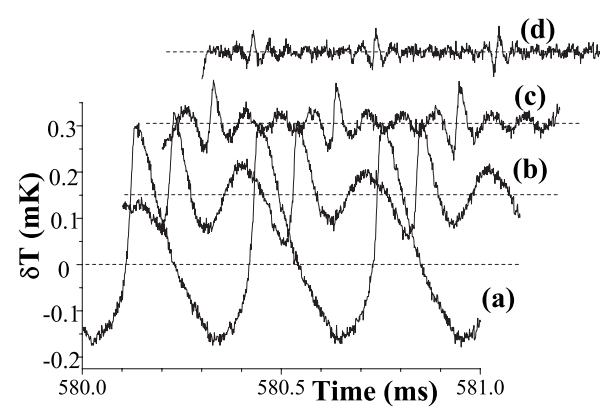

FIG. 2. Second sound wave signal before and after lowfrequency filtration. The cavity was excited at its 33rd resonance with $f_{d}=3227 \mathrm{~Hz}$ and $W=25 \mathrm{~mW} / \mathrm{cm}^{2}$. (a) Original signal. (b) After removal of the main harmonic. (c) With retention of only the 4th and higher harmonics. (d) With retention of only the seventh and higher harmonics.

harmonics and computed the PDF of the rest of the signal. The resultant changes in shape of the signal are shown in Fig. 2, the corresponding changes in its PDF are shown in Fig. 4, and Fig. 3 for those in the filtered spectra.

It is evident that, as expected, the main harmonic is defined by the resonant driving signal and corresponds to a strongly distorted sine wave. The PDF of its main harmonic is similar to that of a sine wave. The driving signal also influences directly the next first harmonics, but fades out for the higher harmonics. Filtration of the low-frequency harmonics transforms a PDF from one that is close to that of a sine wave to the Gaussian-like distribution shown in Fig. 4, which is characteristic of arbitrary wave fields. The intensity of noise in the measurement system was an order of magnitude less than the random fluctuations in second sound intensity. Although the PDF of the signal was approximately Gaussian after removal of the lower harmonics, some asymmetry was visible in all measurements, as shown in Fig. 4. Its origin is evident in the nonsinusoidal shape of the recorded signals as shown, e.g., in Fig. 2(a). The heating peak is accompanied by distinctive breakdowns at the front or tail

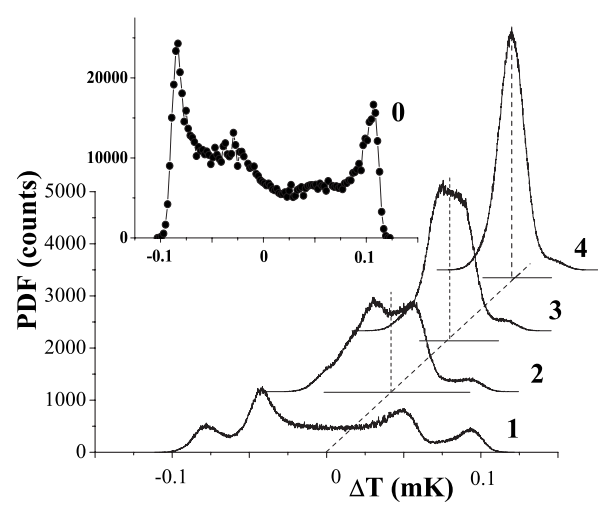

FIG. 3. Evolution of the PDF of a second sound standing wave during sequential removal of the lower frequency harmonics. The number beside each PDF shows the lowest order harmonic that is retained (so that 0 indicates the PDF of the full original signal, 1 indicates retention of second and higher harmonics after first procedure of removal, and so on). The frequencies of the digital cuts are shown in Fig. 1. 


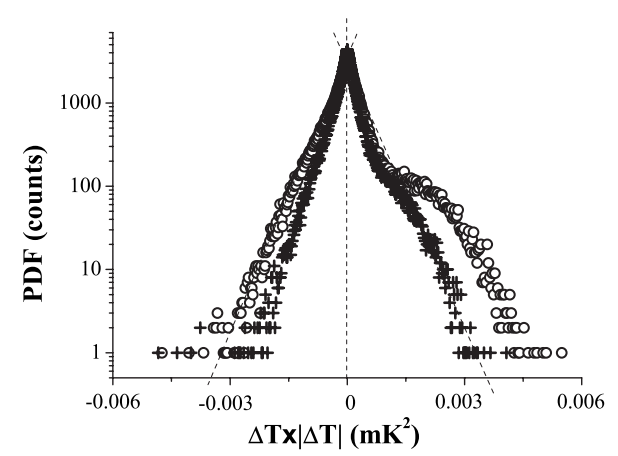

FIG. 4. PDF of the higher harmonics plotted in coordinates chosen to reveal a Gaussian distribution: A logarithmic ordinate and a squared abscissa. A Gaussian would yield two straight lines of equal but opposite slope. The $\bigcirc$ points correspond to cutting frequencies below $15 \mathrm{kHz}$ and the + to cutting below $20 \mathrm{kHz} . f_{d}=3227 \mathrm{~Hz}$, $33 \mathrm{rd}$ resonance, $U_{G}=5 \mathrm{~V}, W=25 \mathrm{~mW} / \mathrm{cm}^{2}$.

depending on the sign of the nonlinear coefficient $\alpha$. Comparable asymmetry was observed for first sound resonances in closed tubes [24], as well as for heating and cooling shock waves in superfluid helium [25] and measurements of the PDF of capillary surface waves [9]. In the present case, because the minima in $\delta T$ are more rounded than the maxima, there is a corresponding asymmetry in the PDF. It decreases for the higher harmonics but does not completely disappear within the experimentally accessible range.

The extent to which the Gaussian distribution is developed corresponds to the extent of development of the turbulent energy cascade: The more developed the energy cascade, the closer the PDF becomes to a Gaussian distribution. Figure 5(a) shows the PDF after removal of the main harmonic (circles), and after removal of both it and the second harmonic (triangles) which clearly brings it closer to being Gaussian in shape. Figure 5(b) for a higher excitation exhibits Gaussian behavior for all harmonics except the main one.

Second sound waves in helium have an approximately linear dispersion ratio $\omega \propto k$. The quasi-one-dimensional longitudinal waves in the resonator have three- and four-wave interactions. The energy transformation to higher frequencies (Kolmogorov-like cascade) is the result of three-wave interaction: $\omega_{d}+\omega_{1} \rightarrow \omega_{2}$, causing the amplitudes of the higher harmonic to increase towards their equilibrium values.

The increasing amplitudes of the higher harmonics leads to intensive interactions between the independent modes within the framework of three-or four-wave processes $\omega_{d}$ $+\omega_{1} \leftrightarrow \omega_{2}$ and $\omega_{d}+\omega_{1} \leftrightarrow \omega_{2}+\omega_{3}$; there will be similar interactions not involving waves at the driving frequency. Such processes will lead to substantial mixing of the phases of the higher frequency modes, which will therefore be less affected by that of the driving force.

We also investigated the effect of increasing the number of wave interactions by adding an additional excitation to the system. Pumping the system by two signals from two separate sine wave generators launches four waves $2 \times f_{1 G}, 2$ $\times f_{2 G}, f_{1 G}-f_{2 G}$, and $f_{1 G}+f_{2 G}$ into the resonator. With pumping only at the 31 st resonance, a developed cascade was formed as shown in Fig. 6(a). The addition of a weak excitation at a lower frequency changes the picture substantially.

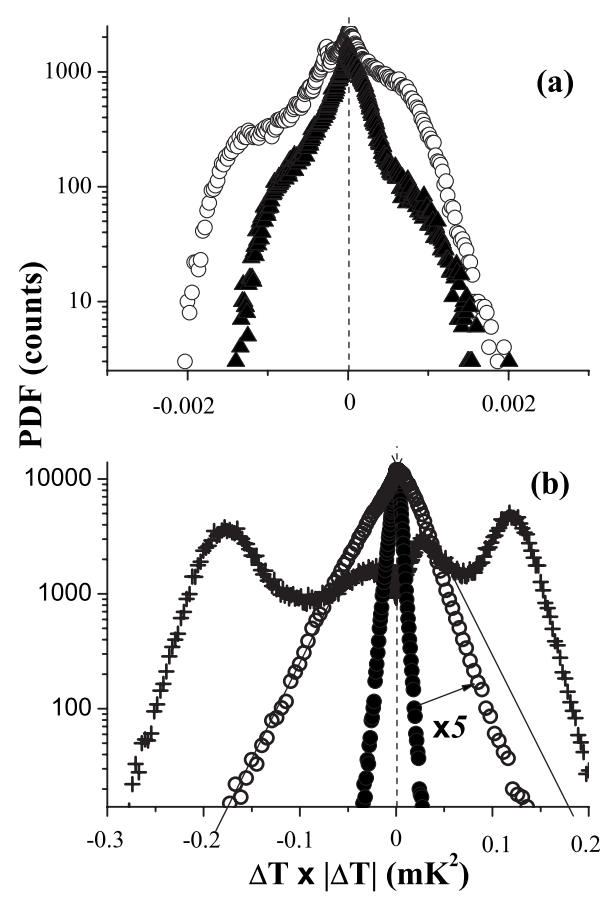

FIG. 5. Increasingly Gaussian behavior as turbulent processes develop. (a) At an excitation of $W=9 \mathrm{~mW} / \mathrm{cm}^{2}$ after removal of the main harmonic $\bigcirc$, and removal of the main and second harmonics A. (b) At $W=25 \mathrm{~mW} / \mathrm{cm}^{2}$. The + are the PDF of the initial signal. The filled circles show the PDF after removal of the main harmonic, and the $\bigcirc$ are the same points with a $5 \times$ abscissa expansion. The cavity was driven at its 11 th resonance with $f_{d}=1060 \mathrm{~Hz}$.

The interactions of the resultant four waves give rise to numerous combinational signals, as shown in Fig. 6(b). Furthermore, interactions between the main and combinational waves lead to attenuation of the developed turbulent cascade and change the distribution function within the inertial interval [26]. Suppression of the main turbulent cascade is indicated by a change in the frequency dependence of the spectral maxima from $A \propto f^{-1.79}$ in (a) to $A \propto f^{-2.18}$ in (b). This phenomenon is connected with a redistribution of the energy

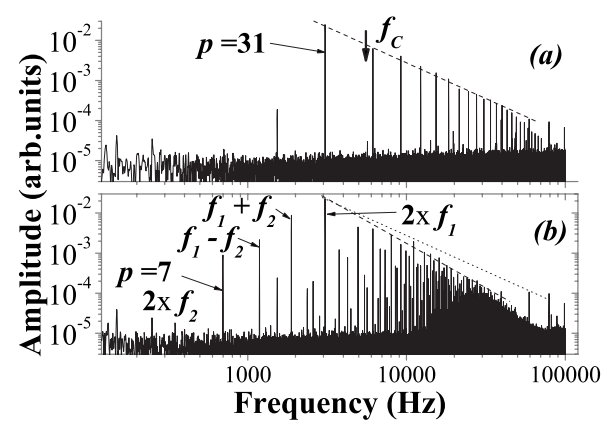

FIG. 6. Power spectra of second sound signals showing turbulent cascades formed while pumping at one forcing frequency (upper figure) and double excitation with two forcings (lower figure). The main forcing was at the 31 st resonance $\left(U_{1}=7 \mathrm{~V} ; W\right.$ $\left.=49 \mathrm{~mW} / \mathrm{cm}^{2}\right)$ and the additional perturbation at the seventh resonances $\left(U_{2}=2 \mathrm{~V} ; W=4 \mathrm{~mW} / \mathrm{cm}^{2}\right)$. The arrow indicates the position of the high-pass filter for both parts of the figure. The positions of the main combination frequencies are indicated. 


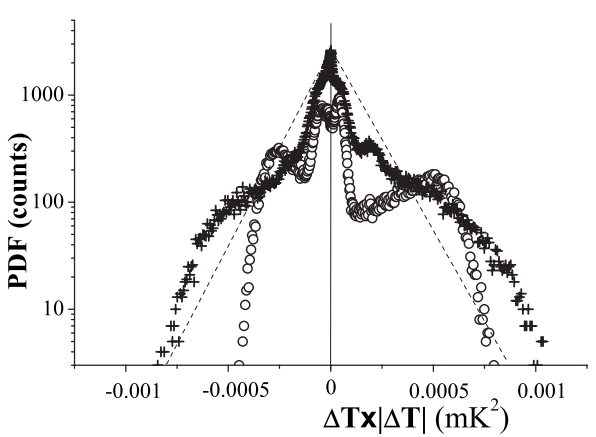

FIG. 7. Effect on the PDF of a second sound signal of adding a second weak forcing. The high-frequency band-pass filter removed frequencies below $f_{C}=5900 \mathrm{~Hz}$, thus eliminating the fundamental frequency of the main turbulent cascade as illustrated in Fig. 6. The $\bigcirc$ points correspond to single forcing, and the + to double forcing. The main forcing was at $f_{d}=3067.4 \mathrm{~Hz}$ (31st resonance), $W$ $=49 \mathrm{~mW} / \mathrm{cm}^{2}$; and the lower frequency perturbation was applied at $f_{d}=698 \mathrm{~Hz}$ (seventh resonance), $W=4 \mathrm{~mW} / \mathrm{cm}^{2}$. The spectra of the signals are shown in Fig. 6

flux between a large number of additional interacting combinational modes. A similar effect was observed for the system of surface waves on liquid hydrogen [27].

The appearance of a set of combinational waves, and the increasing number of interactions between the different harmonics, lead to the formation of a Gaussian-like PDF in the higher spectral range. The result of cutting the spectrum of the signal below $f_{c}=5900 \mathrm{~Hz}$ is shown in Fig. 7. Note that the frequency cut (arrows in Fig. 6) is made below the second harmonic of the developed cascade with a single excitation signal.

Finally, we enter two caveats in relation to the above discussion. First, we note that the resonant frequency of the forcing signal differs slightly from the frequency of free oscillation due to the presence of the small heat flux from the heater [28]. Thus, the wavelength of the driving signal at resonance is not exactly equal to the wavelength of the traveling wave corresponding to the eigenfrequency in the cavity and the integer length of higher harmonics. Correspondingly, the influence of the phase of the driving force diminishes in the higher harmonics. The effect is clearly visible for our so-called "eleventh resonance" (Fig. 5), which we suspect is coupled to the first radial resonance (see above): The frequencies of the fundamental radial mode and higher radial modes are incommensurate. Second, the differing velocities corresponding to the different amplitudes of the harmonics in the cascade mean that the phases and resonant frequencies of the different modes are slightly different from those expected.

\section{CONCLUSIONS}

The main conclusion is that the removal of low-frequency components in the signal from turbulent second sound in our resonator yields probability densities with Gaussian-like distributions: With successive removal of the lower harmonics, the strong influence of the driving signal (with cophasal behavior) weakens, and the PDF changes from the shape corresponding to a sine wave towards a Gaussian distribution, representing the statistical average of random multiple harmonics. It was already known that the Kolmogorov-like power-frequency distribution arising from a high-intensity pumping signal takes the form $A_{i} \propto \omega^{-n}$, where $n \sim 1.5$. More intense driving yields a lower value of the power $n$, a longer inertial interval, and relatively larger amplitude of higher harmonics [23]. We have also found that, under some circumstances, an inverse energy cascade can exist, carrying wave energy towards lower frequencies [29]. We have now shown that, although the driving signal exerts a strong influence on the first few harmonics in the resonator, this influence quickly dies out in the higher harmonics where the PDFs become nearly Gaussian, apparently corresponding to chaoticlike behavior. Such phenomena are to be anticipated in wave turbulent systems quite generally but, to our knowledge, have not previously been demonstrated experimentally.

Pumping the system with an additional weak excitation leads to the formation of numerous combinational waves, and a partial suppression of the main energy cascade. The interaction between the combinational waves and the main driving signal shifts the region with a Gaussian-like PDF towards lower frequencies. Thus, the form of acoustic turbulence revealed by the experiments apparently exhibits the features of both strong wave interaction and weak turbulence.

\section{ACKNOWLEDGMENTS}

We acknowledge valuable discussions with G. V. Kolmakov, A. A. Levchenko, E. A. Kuznetsov, V. Krasnosselskikh, V. S. L'vov, V. V. Lebedev, and L. P. Mezhov-Deglin. The work was supported by Engineering and Physical Sciences Research Council (UK) and by the Royal Society of London.
[1] L. F. Richardson, Weather Prediction by Numerical Process (Cambridge University Press, Cambridge, 1922).

[2] V. Zakharov, G. Falkovich, and V. L'vov, Kolmogorov Spectra of Turbulence I (Springer, Berlin, 1992).

[3] V. E. Zakharov and N. N. Filonenko, J. Appl. Mech. Tech. Phys. 8, 37 (1967).

[4] A. N. Kolmogorov, C. R. (Dokl.) Acad. Sci. URSS 31, 301 (1941).
[5] A. N. Kolmogorov, C. R. (Dokl.) Acad. Sci. URSS 31, 538 (1941).

[6] V. E. Zakharov, O. A. Vasilyev, and A. I. Dyachenko, JETP Lett. 73, 68 (2001).

[7] I. P. D. De Silva and H. J. S. Fermando, Phys. Fluids 6, 2455 (1994).

[8] E. Henry, P. Alstrom, and M. T. Levinsen, Europhys. Lett. 52, 27 (2000). 
[9] E. Falcon, C. Laroche, and S. Fauve, Phys. Rev. Lett. 98, 094503 (2007).

[10] B. B. Kadomtsev and V. I. Karpman, Sov. Phys. Usp. 103, 193 (1971).

[11] S. N. Gurbatov, A. I. Saichev, and G. Yakushkin, I, Sov. Phys. Usp. 141, 221 (1983).

[12] J. M. Burgers, Proc. R. Acad. Sci. Amsterdam 43, 2 (1940).

[13] V. B. Efimov, G. V. Kolmakov, A. S. Kuliev, and L. P. Mezhov-Deglin, Fiz. Nizk. Temp. 24, 81 (1998).

[14] Weinan E and E. Vanden Eijnden, Phys. Rev. Lett. 83, 2572 (1999).

[15] O. V. Rudenko, Sov. Phys. Usp. 149, 413 (1986).

[16] O. V. Rudenko, Sov. Phys. Usp. 163, 1011 (1995).

[17] A. J. Dessler and W. M. Fairbank, Phys. Rev. 104, 6 (1956).

[18] I. Y. Borisenko, V. B. Efimov, and L. P. Mezhov-Deglin, Fiz. Nizk. Temp. 14, 1123 (1988).

[19] V. B. Efimov, A. N. Ganshin, P. V. E. McClintock, G. V. Kolmakov, and L. P. Mezhov-Deglin, J. Low Temp. Phys. 145, 155 (2006).

[20] M. Giltrow, M. J. Boylett, N. S. Lawson, A. Hammiche, O. J. Griffiths, J. K. Wigmore, and V. B. Efimov, Meas. Sci. Tech- nol. 14, N69 (2003).

[21] H. Davydowitz, Y. L'vov, and V. Steinberg, Physica D 84, 635 (1995).

[22] V. B. Efimov, A. N. Ganshin, P. V. E. McClintock, G. V. Kolmakov, and L. P. Mezhov-Deglin, J. Low Temp. Phys. 148, 251 (2007).

[23] G. V. Kolmakov, V. B. Efimov, A. N. Ganshin, P. V. E. McClintock, and L. P. Mezhov-Deglin, Phys. Rev. Lett. 97, 155301 (2006).

[24] W. Chester, J. Fluid Mech. 18, 44 (1964).

[25] G. V. Kolmakov, V. B. Efimov, A. S. Kuliev, and L. P. Mezhov-Deglin, Czech. J. Phys. 46, S1, 83 (1996).

[26] P. V. E. McClintock, V. B. Efimov, A. N. Ganshin, G. V. Kolmakov, and L. P. Mezhov-Deglin, J. Low Temp. Phys. 150, 394 (2008).

[27] L. V. Abdurakhimov, M. Yu. Brazhnikov, and A. A. Levchenko, J. Low Temp. Phys. 150, 431 (2008).

[28] D. Greywall and G. Ahlers, Phys. Rev. A 7, 2145 (1973).

[29] A. N. Ganshin, V. B. Efimov, G. V. Kolmakov, L. P. MezhovDeglin, and P. V. E. Mcclintock, Phys. Rev. Lett. 101, 065303 (2008). 